\title{
On-Demand Spectrum and Space Defragmentation in an Elastic SDM/FDM/TDM Network with Mixed Multi- and Single-core Fiber Links
}

\author{
N. Amaya ${ }^{1 *}$, M. Irfan ${ }^{1}$, G. S. Zervas ${ }^{1}$, R. Nejabati ${ }^{1}$, D. Simeonidou ${ }^{1}$, \\ V. J. F. Rancaño ${ }^{2}$, F. Parmigiani' ${ }^{2}$, P. Petropoulos' ${ }^{2}$, D. J. Richardson ${ }^{2}$, \\ J. Sakaguchi ${ }^{3}$, W. Klaus ${ }^{3}$, B.J. Puttnam ${ }^{3}$, T. Miyazawa ${ }^{3}$,Y. Awaji ${ }^{3}$, N. Wada ${ }^{3}$, I. Henning ${ }^{4}$ \\ (1) High-performance Networks Group, University of Bristol, United Kingdom, *namayago@hotmail.com \\ (2) Optoelectronics Research Centre, University of Southampton, Southampton, SO17 1BJ, UK \\ (3) National Institute of Information and Communications Technology (NICT), Tokyo, Japan
} (4) University of Essex, Colchester, Essex, United Kingdom

\begin{abstract}
We show on-demand multi-wavelength spectrum and space defragmentation in an SDM and elastic network with four programmable nodes and two multi-core fiber links. The combined approach is shown to reduce blocking and hardware requirements in small nodes.

OCIS codes: (060.4250) Networks; (060.4510) Optical Communications.
\end{abstract}

\section{Introduction}

Elastic optical networking (EON) has been proposed as a viable approach to efficiently support high-speed optical channels beyond $100 \mathrm{~Gb} / \mathrm{s}$ [1]. EON allows to accommodate high-speed channels with arbitrary bandwidth requirements, e.g. $400 \mathrm{G}, 1 \mathrm{~T}$ and beyond, as well as to increase spectral efficiency for low bitrate channels, e.g. 10G, by allocating spectrum in a flexible manner and depending on channel requirements [2]. However, in a dynamic network environment, where optical channels are constantly added and removed, elastic spectrum allocation may give rise to spectrum fragmentation, which increases the blocking probability. Several techniques have been proposed to defragment the spectrum, such as wavelength conversion [2] and continuous tuning of the transmitted wavelength [3]. However, most of the techniques considered to date relocate only a single wavelength at a time with a specific polarization, bitrate and modulation format. To make this process more efficient it is necessary to develop a multi-wavelength defragmentation technique that is polarization, bitrate and modulation format independent.

Meanwhile, Space Division Multiplexing (SDM) has been proposed to increase optical fibre transmission capacity by increasing the number of spatial channels over a single fibre [4]. However, successful deployment of networks with SDM links will require scalable and flexible optical nodes able to support granularities consistent with traffic switching requirements while minimizing hardware requirements. We have recently proposed an adaptable optical node that provides a wide range of granularities and supports SDM requirements [5]. High scalability is achieved by switching traffic at coarse granularities, e.g. fibre-to-core, core-to-core, core-to-fibre. Thus,

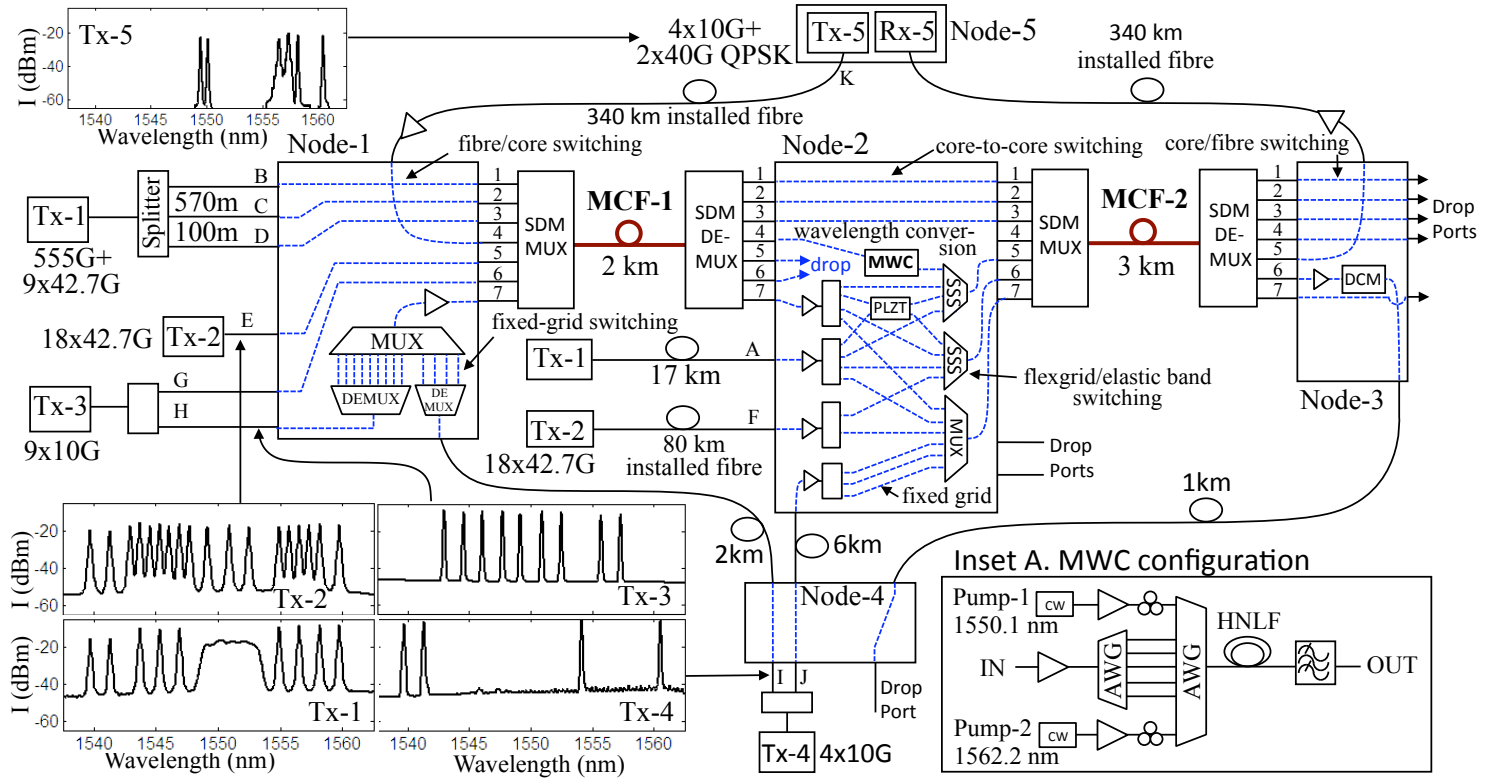

Figure 1. Experimental network configuration. Inset A: FWM-based wavelength converter schematic. 
relatively small nodes can switch large volumes of traffic. However, switching at fiber/core granularities requires all channels in the same fibre/core input to go to the same fibre/core output, i.e. space defragmentation.

In this paper, we propose and experimentally demonstrate on-demand polarization-independent multiwavelength spectrum defragmentation, as well as space defragmentation, in an elastic network with four programmable nodes and two multi-core fiber links. The proposed spectrum defragmentation approach simultaneously wavelength-converts one $40 \mathrm{~Gb} / \mathrm{s}$ QPSK and two $10 \mathrm{~Gb} / \mathrm{s}$ OOK channels, thereby reducing blocking due to wavelength contention. In turn, space defragmentation, performed in a large node, is shown to alleviate switching requirements in a smaller node, thereby facilitating the provisioning of end-to-end services.

\section{Elastic multigranular network configuration}

The network scenario, shown in Fig. 1, represents a future elastic and dynamic transparent network, with programmable optical nodes of different sizes and capabilities, linked by a mix of multi-core and single mode fiber (SMF). Nodes 1, 2 and 3 are connected by two 7-core fibre sections of 2-km (MCF-1) and 3-km (MCF-2). Both MCFs connect to optical nodes using SDM MUX/DEMUX devices based on free-space optics [6]. The average loss of the MCF combined with SDM MUX/DEMUX was measured to be $2 \mathrm{~dB}$ and $2.4 \mathrm{~dB}$ for MCF- 1 and MCF-2 respectively. The average measured cross-talk (including the contributions of MUX / DEMUX) was $-56.5 \mathrm{~dB}$ and $53.8 \mathrm{~dB}$ for MCF-1 and MCF-2 respectively. Node-4 is connected to Nodes 1, 2 and 3 by different lengths of SMF, as illustrated in Fig. 1. Node- 5 is remotely located and connects to Nodes 1 and 3 through $340-\mathrm{km}$ dispersion compensated dark fibre links. Nodes 1-4 are based on the Architecture-on-Demand (AoD) concept [5] and consist of an optical backplane that interconnects MCF/SMF fibre inputs, functional modules and MCF/SMF fibre outputs. The backplanes of Nodes 1, 2 and 4 are implemented as independent partitions of a 160x160 3D-MEMS optical switch with a $20-\mathrm{ms}$ switching time and an average loss of $2 \mathrm{~dB}$ per cross-connection. Node- 3 is implemented with a $16 \times 16$ beam-steering switch [7] with an average loss of $0.59 \mathrm{~dB}$ per cross-connection. The modulation-, bit rate- and polarization-independent multi-channel spectrum defragmentation is achieved by wavelength conversion based on four-wave mixing (FWM) in a highly nonlinear fiber (HNLF) pumped by two orthogonally polarized cw lasers, see inset of Fig.1. The pumps are located at the two extremes of the signal/idler bandwidth and coupled together with the signals via arrayed waveguide gratings (AWGs), as illustrated in Fig. 1 inset A.

\section{Spectrum and space defragmentation}

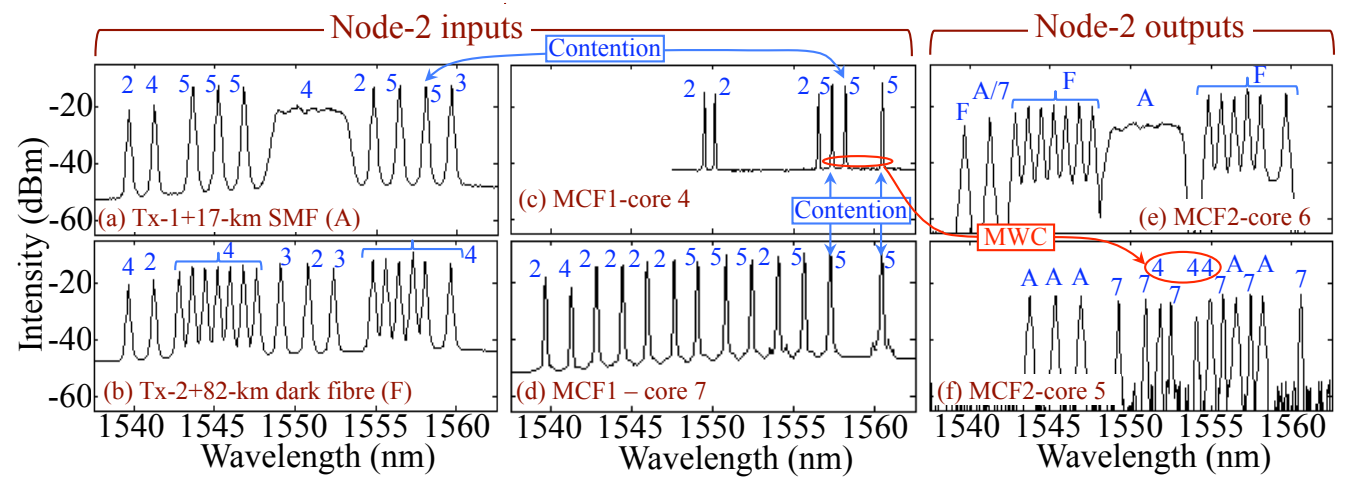

Figure 2. Optical spectrum plots at relevant Node-2 inputs and outputs.

Figures 2 (a)-(d) show spectrum plots at relevant Node-2 inputs with channels labeled according to their destination node. Figures 2 (e)-(f) show relevant Node-2 outputs. Output channel labels represent the corresponding Node-2 input. Three of the wavelengths in MCF-1 core 4 (Fig. 2c) present contention with one channel from SMF input A (Fig. 2a) and two channels from MCF-1 core 7 (Fig. 2d). These channels require being transmitted over Node-3, and $340-\mathrm{km}$ of installed SMF, to reach their destination (Node-5). It is not possible to resolve this contention using the space dimension, due to the single-core fiber between Nodes 3 and 5. Also, Node-3 is not provisioned with wavelength conversion or (de)multiplexing capabilities. Therefore, Node-2 is programmed to connect the polarization-independent multi-channel converter to input MCF-1 core 4, as shown in Fig. 1. Thus, the contending channels are relocated by means of simultaneous multi-wavelength conversion (MWC) and aggregated with all the other channels that go to Node-5 on MCF-2 core 5, as shown in Fig. 2(f). As the channels now aggregated in MCF-2 core 5 have a common destination, i.e. Node-5, channel (de)multiplexing is not required for switching them at Node3 , as shown in Fig. 1. Similarly, channels destined to Node-4 are aggregated at the output of Node-2 in MCF-2 core 6, as shown in Fig. 2(e), thereby obviating the need for (de)multiplexing at Node-3. Therefore, performing spectrum and space defragmentation in Node-2 alleviates channel (de)multiplexing requirements in Node-3. 


\section{Channel performance}

Figure 3 (a) shows bit error ratio (BER) plots and constellation diagrams for the phase modulated channels as a function of optical signal to noise ratio (OSNR), which were sent and received at Node- 5 after going through Node1, MCF-1, Node-2 (with WC), MCF-2 and Node-3. BER results for the $10 \mathrm{~Gb} / \mathrm{s}$ OOK channels, originated and received at Node-5 after going through Node-1, MCF-1, Node-2 (with WC), MCF-2 and Node-3, are shown in Fig. 3 (b). At BER $=10^{-9}$, end-to-end OSNR penalties of $2 \mathrm{~dB}$ and $2.8 \mathrm{~dB}$ are observed for the converted $1551.8 \mathrm{~nm}$ and $1554 \mathrm{~nm}$ wavelengths respectively. Figure 3 (c) shows typical BER curves for channels ending at Node-4. End-toend penalties of $1.8 \mathrm{~dB}, 2.7 \mathrm{~dB}$ and $3.7 \mathrm{~dB}$ are observed for the $555 \mathrm{~Gb} / \mathrm{s}$ (sub-carrier 25), $42.7 \mathrm{~Gb} / \mathrm{s}$, and $42.7 \mathrm{~Gb} / \mathrm{s}$ sub-wavelength channels respectively. Figure 3 (d) shows back-to-back and end-to-end sensitivities of the
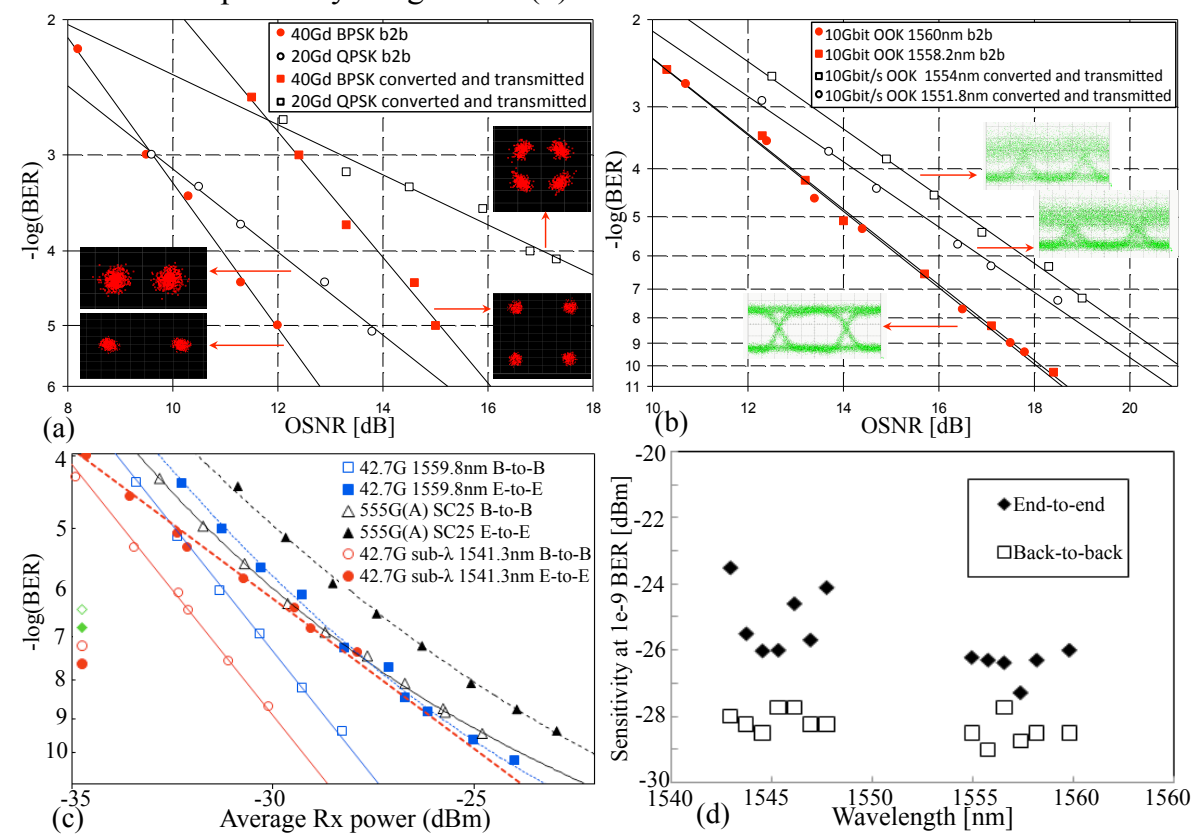

Figure 3. BER measurements at various points in the network.

continuous $42.7 \mathrm{~Gb} / \mathrm{s}$ channels received at Node- 4 . The highest penalty, of $4.5 \mathrm{~dB}$ was measured for the channel at $1542.9 \mathrm{~nm}$ due to the lower channel power and OSNR, as shown in Fig. 2(e). Note that power equalization was not performed at the outputs of Node-2.

\section{Conclusion}

We have proposed and experimentally demonstrated on-demand polarization-independent multi-wavelength spectrum defragmentation, as well as space defragmentation, in an elastic SDM/FDM/TDM network with four programmable nodes and two multi-core fiber links. The proposed multi-channel spectrum defragmentation approach was implemented in a programmable node to perform on-demand waveband conversion and resolve issues of wavelength contention. Space defragmentation was shown to alleviate switching requirements of smaller nodes, thereby facilitating the provisioning of end-to-end services. BER results showed error-free performance for the OOK channels and a good margin below the $2 \times 10^{-3} \mathrm{FEC}$ threshold for the $555 \mathrm{~Gb} / \mathrm{s}, 40 \mathrm{G}$ BPSK and 40G QPSK channels.

\section{Acknowledgements}

This work is supported by the EPSRC grant EP/I01196X: The Photonics Hyperhighway. The authors are grateful to Mitsubishi Cable Industries from Japan for providing the MCFs and would like to thank Polatis for the loan of the low-loss space switch and Yenista Optics for the bandwidth-variable optical filter.

\section{References}

[1] O. Gerstel et al., Coms. Mag., vol. 50, no. 2, pp. s12 -s20, february 2012.

[2] N. Amaya et al., ECOC 2011, TH.13.K.1 (2011).

[3] K. Sone et al., ECOC 2012, Th.3.D.1. (2012).

[4] Jun Sakaguchi et al., OFC 2012, PDP5C.1, (2012).

[5] N. Amaya et al., ECOC 2012, Th.3.D.3C (2012).

[6] W. Klaus et al., IEEE Summer Topicals 2012, WC3.3

[7] M. Bitting, AUTOTESTCON 2004. 